\title{
Téoros
}

Revue de recherche en tourisme

\section{Le tourisme social face à l'avenir touristique européen}

\section{André Hut}

Volume 9, numéro 3, novembre 1990

URI : https://id.erudit.org/iderudit/1079890ar

DOI : https://doi.org/10.7202/1079890ar

Aller au sommaire du numéro

Éditeur(s)

Université du Québec à Montréal

ISSN

0712-8657 (imprimé)

1923-2705 (numérique)

Découvrir la revue

Citer cet article

Hut, A. (1990). Le tourisme social face à l'avenir touristique européen. Téoros, 9(3), 38-39. https://doi.org/10.7202/1079890ar d'utilisation que vous pouvez consulter en ligne.

https://apropos.erudit.org/fr/usagers/politique-dutilisation/ 
EUR-INFO

Le tourisme social face à

André Hut* I'avenir touristique européen

Les douze pays regroupés dans la Communauté économique européenne (C.E.E.) ont décidé d'ouvrir, en 1993, le marché intérieur qu'ils constituent, avec 320 millions de consommateurs, a la libre circulation des capitaux, des marchandises et des personnes. L'étape suivante sera l'union politique et monétaire. Dès à présent, la décision de l'ouverture (frontières) du marché intérieur provoque pas mal de changements au niveau des entreprises qui, par des regroupements, OPA etconcentrations, veulent se positionner favorablement face â la concurrence. Cela vaut également dans le domaine des entreprises touristiques de transport, d'agences de voyages, d'hôtellerie et des entreprises immobilières touristiques. Le volet social, de son côté, est moins avancé et la "charité sociale" de la C.E.E. souffre d'une gestation lente et difficile, digne d'un eléphant blanc.

Il est réjouissant, dans ce contexte, de faire connaitre, afin de l'apprécier à sa juste valeur, 1 ' initiative prise par le Bureau international du tourisme social (BITS), d'organiser, du 16 au 19 mai 1990, à Rome, sa "Deuxième Conférence européenne du tourisme social et de la jeunesse" at l'occasion de l'Année européenne du tourisme proclamée pour 1990 par la Communauté économique européenne. Il faudra d'ailleurs revenir ultérieurement sur les positions, les politiques et les actions des diverses instances européennes impliquées dans le développement du tourisme sur le continent ainsi que sur les objectifs et le bilan de cette Année européenne du tourisme organisée par la C.E.E..

Enfin, la Déclaration finale rédigée et proclamée par le secrétaire général du BITS, Raymond Stélandre - reprise in extenso cidessous - cadre assez bien avec le thème de ce numéro de Téoros en resituant les objectifs, la place et la matière du tourisme associatif, social et non-marchand dans le secteur de la distribution au sein du marché touristique ${ }^{(1)}$.
Expert en tourisme, professeur et animateur, monsieur André Hut est correspondant de Tabros en Europe.
Pour un tourisme social et de la jeunesse dans l'Europe de demain

Déclaration finale par

M. Raymond Stélandre

Secrétaire général du BITS

C'est à Rome, siège historique du Traité de base de la communauté européenne que s'est tenue la 2ème Conférence européenne du tourisme social et de la jeunesseàl'occasion de l'Année européenne du tourisme.

Au delà du geste symbolique, la signification profonde de cette démarche est de situer le tourisme social et de la jeunesse à la place qui lui revient parmi les objectifs de développement de la communauté européenne.

Àla veille du grand marché communautaire de 1993, le tourisme apparaît comme un élément majeur, source de développement économique et porteur d'enrichissement humain et culturel.

Profondément enraciné dans la réalité humaine et sociale européenne, le tourisme social et de la jeunesse est porteur des attentes et des espoirs des larges couches de la population, en particulier des moins favorisés aux plans économique et culturel et des jeunes qui incarnent la réalité européenne des années 2000.

Partie intégrante de l'économie sociale, le tourisme social et de la jeunesse des douze pays de la communauté n'a, aujourd'hui et demain, de vraie dimension qu'européenne. C'est à cette dimension qu'ilentend hisser toute son action.

Ce faisant il est convaincu d'être un facteur important de construction de l'Europe des citoyens, objectif ultime de la communauté européenne.

Traduisant sa volonté de progresser concrètement et systématiquement dans cette voie, la 2ème Conférence 
européenne du tourisme social et de la jeunesse met l'accent sur trois objectifs précis pour les prochaines années. Il est évident que ces objectifs ne résument pas à eux seuls toutes les préoccupations du tourisme social et de la jeunesse. Dans la sphère, et avec les méthodes qui leur sont propres, les organisations nationales et internationales poursuivent le développement du tourisme social et de la jeunesse. Leur action vise à mettre tout le tourisme, les loisirs et la culture à la portée de tous, jeunes et moins jeunes, quelle que soit leur situation économique, sociale ou culturelle.

\section{1- Pour un chèque vacances loisirs européen}

Il importe de s'interroger sur les voies et moyens susceptibles de réduire les inégalités qui marquent encore la pratique de l'activité touristique, cette forme de liberté à laquelle aspirent tous les hommes.

La communauté européenne et les pays de I'A.E.L.E. ont interêt à favoriser le développement touristique tant du point de vue social qu'économique.

L'un des moyens répondantà cet objectif est l'instauration d'ụn chèque vacances loisirs européen. A partir des expériences suisse et française, il s'agit de mettre en chantier une étude pan-européenne en vue de l'extension de la formule à l'ensemble des pays européens. Cette opération, appuyée dans chaque pays par un comité pour le cheque vacances, doit permettre d'aboutir à la mise en place dans chaque pays d'organes d'émission et de gestion ainsi qu'à la constitution d'un organe européen de coordination, une charte européenne des chèques vacances constituant le cadre juridique de référence.

\section{2- Pour un réseau européen de villages de vacances}

Dans plusieurs pays, des installations de tourisme social - maisons, centres et villages de vacances - ont été créés avec l'appui des pouvoirs publics à l'initiative ou avec la collaboration de mouvements sociaux et/ou d'organismes de tourisme social, tantà destination des familles que des jeunes.

Ces réseaux d'installations contribuent à faciliter l'accès du plus grand nombre au tourisme. Al'heure de l'Europe, ces installations ne peuvent dorénavant se concevoir qu'à la dimension européenne, première étape de la dimension internationale qui définit, par nature, le tourisme social.

Il s'agit, d'une part, d'européaniser les installations existantes en organisant la multiplication des échanges bi- ou multilatéraux et en labelisant les installations qui répondent aux exigences du tourisme social européen. D'autre part, I'heure est venue de développer des réseaux d'installations européennes intégrées.

L'expérience d'Eurovillage, commencée au Cap d'Agde par une collaboration franco-belge et poursuivie par le G.E.I.E. "Eurovillages" constitué actuellement par des associations française, belge, italienne, suisse et allemande, mérite d'être étendue et appuyée.

Cette initiative non exclusive ouvre la voie à d"autre projets européens intégrés qui méritent également développement et appui.

\section{3- Pour une formation des jeunes aux métiers du tourisme}

Le tourisme constitue déjà aujourd'hui et constituera davantage encore dans les années à venir un des secteurs principaux de l'activité socio-économique de l'Europe de demain.

L'importance de la formation des jeunes aux métiers du tourisme apparaît dès lors comme un objectif majeur qui rencontre les interrogations des jeunes et des responsables sociaux quant aux perspectives de l'emploi.

Cette formation, si elle comporte un tronc commun à l'ensemble de l'activité touristique, doit impérativement tenir compte de la spécificité du tourisme social qui exige à la fois des qualités militantes et des qualités professionnelles.

Un inventaire européen des besoins et des programmes s'impose en vue de définir un système de formation adapté aux exigences futures, tenant compte à la fois des particularités nationales et de la dimension européenne. L'expérimentation nationale et la coopération européenne sont complémentaires.
II y va autant de l'avenir des jeunes concernés par les métiers du tourisme que de l'avenir même de la qualité du tourisme en général et du tourisme social en particulier.

\#\#*\#*

Afin de poursuivre la réalisation de ces objectifs, la 2ème Conférence européenne du tourisme social et de la jeunesse en appelle

- auxassociations et organismes de tourisme social et de la jeunesse des pays européens. Ce sont les premiers acteurs des initiatives à prendre dans les différents domaines envisagés. Demain comme hier, l'avenir du tourisme social dépendra d'abord de leur dynamismeet de leur capacité d'entreprise.

- aux mouvements sociaux et aux pouvoirs publics régionaux et nationaux dontl'appui est nécessaire au développement et aux adaptations du tourisme social.

- aux instances de la Communauté européenne initiatrices de l'Année européenne du tourisme à laquelle le tourisme social et de la jeunesse est intimement associé.

Les trois objectifs précis qui font l'objet des conclusions de cette conférence appellent le concours des Communautés, notamment au plan financier, institutionnel et juridique.

C'est ensemble, chacun à notre niveau, que, par le tourisme social, nous construisons l'Europe des citoyens.

Notes explicatives

(1) Le compte rendu complet de la 2 eme Confórence europtenne du touriame et de la jeuneses" "Rome, 16-19 mai 1990 peut ötro obtenu au Secreteriat du BITS, rue de la Loi, 63 - B 1000 Bruselles - Belgique. II est disponible au Centre d'études du tourisme. 515 ouest, rue Ste-Catherine, be ótage. Tél.: $|514|$ $987-8516$. 